University of Nebraska - Lincoln

DigitalCommons@University of Nebraska - Lincoln

Hendrik J. Viljoen Publications

Chemical and Biomolecular Research Papers --

Faculty Authors Series

$5-1-2009$

\title{
A kinetic study of in vitro lysis of Mycobacterium smegmatis
}

William J. Valente

University of Nebraska - Lincoln, wvalente4@unl.edu

Elsje Pienaar

University of Nebraska - Lincoln, epienaar2@unl.edu

A. Fast

University of Nebraska - Lincoln

A. Fluitt

University of Nebraska - Lincoln

Scott E. Whitney

University of Nebraska - Lincoln, swhitney4@unl.edu

See next page for additional authors

Follow this and additional works at: https://digitalcommons.unl.edu/cbmeviljoen

Part of the Chemical Engineering Commons

Valente, William J.; Pienaar, Elsje; Fast, A.; Fluitt, A.; Whitney, Scott E.; Fenton, Robert J.; Barletta, Raul G.; Chacon, O.; and Viljoen, Hendrik J., "A kinetic study of in vitro lysis of Mycobacterium smegmatis " (2009). Hendrik J. Viljoen Publications. 12.

https://digitalcommons.unl.edu/cbmeviljoen/12

This Article is brought to you for free and open access by the Chemical and Biomolecular Research Papers -- Faculty Authors Series at DigitalCommons@University of Nebraska - Lincoln. It has been accepted for inclusion in Hendrik J. Viljoen Publications by an authorized administrator of DigitalCommons@University of Nebraska - Lincoln. 


\section{Authors}

William J. Valente, Elsje Pienaar, A. Fast, A. Fluitt, Scott E. Whitney, Robert J. Fenton, Raul G. Barletta, 0. Chacon, and Hendrik J. Viljoen 
Published in Chemical Engineering Science 64:9 (May 1, 2009), pp. 1944-1952; doi 10.1016/j.ces.2008.12.015

Copyright (c) 2008 Elsevier Ltd. Used by permission. http://www.elsevier.com/locate/ces

Submitted November 3, 2008; revised December 12, 2008; accepted December 17, 2008; published online December $25,2008$.

\title{
A kinetic study of in vitro lysis of Mycobacterium smegmatis
}

\author{
W. J. Valente, ${ }^{1}$ E. Pienaar, ${ }^{1}$ A. Fast, ${ }^{1}$ A. Fluitt, ${ }^{1}$ S. E. Whitney, ${ }^{1}$ R. J. Fenton, ${ }^{2}$ R. G. Barletta, ${ }^{2}$ O. \\ Chacon, ${ }^{2,3}$ and H. J. Viljoen ${ }^{1}$ \\ ${ }^{1}$ Department of Chemical and Biomolecular Engineering, University of Nebraska-Lincoln, NE 68588-0643, USA \\ ${ }^{2}$ Department of Veterinary \& Biomedical Sciences, University of Nebraska-Lincoln, NE, USA \\ ${ }^{3}$ Seccion de Bacteriologia, Corporacion para Investigaciones Biologicas (CIB), Carrera 72A No. 78B 141, A.A. 7378, Medellín, Colombia \\ Corresponding author - H. J. Viljoen, tel 402 472-3331, fax 402 472-6989, email hviljoen1@unlnotes.unl.edu
}

\begin{abstract}
The traditional diagnostic tests for tuberculosis consist of an acid fast stain and a culture test from a sputum sample. With the emergence of drug resistant strains of tuberculosis, nucleic acid amplification has become the diagnostic test of choice. The nucleic acid amplification test consists of four steps: sputum sample collection, lysis of bacilli to release DNA, DNA amplification by PCR and detection of PCR products. The DNA extraction step has been largely overlooked and this study describes a systematic approach to measure the kinetics of cell lysis in a Tris-EDTA buffer. Mycobacterium smegmatis is a saphorytic, fast-growing mycobacterium that is often used as a surrogate of Mycobacterium tuberculosis in laboratory studies. M. smegmatis cells have been transformed with green fluorescent protein (GFP) genes. Transformed cells are lysed in a temperature-controlled cuvette that is equipped with optical input/output. The fluorescence signal increases when the GFP is released from lysed cells, and the extent of lysis of the loaded cells can be followed in real time. The experimental results are complemented by two theoretical models. The first model is based on a Monte Carlo simulation of the lysis process and the accompanying probability density function, as described by the Fokker-Planck equation. The second model follows a chemical reaction engineering approach: the cell wall is modeled as layers, where each layer is made up of "blocks". Blocks can only be removed if they are exposed to the lysis solution and the model describes the rate of block exposure and removal. Both models are consistent with the experimental results. The main findings are: (1) the activation energy for M. smegmatis lysis in Tris-EDTA buffer is $22.1 \mathrm{kcal} / \mathrm{mol}$, (2) cells lyse on the average after 14-17\% loss in cell wall thickness locally, (3) with the help of the models, the initial distribution in cell wall thickness of the population can be resolved and (4) near complete lysis of the cells is accomplished in $200 \mathrm{~s}$ at $80{ }^{\circ} \mathrm{C}$ $\left(90 \mathrm{~s}\right.$ at $90^{\circ} \mathrm{C}$ ). The results can be used to design an optimal lysis protocol that compromises between shorter processing times at higher temperature and reduced thermal damage to DNA at lower temperature.
\end{abstract}

Keywords: kinetics, lysis, Mycobacterium smegmatis, mathematical modeling, molecular biology, Fokker-Planck

\section{Introduction}

The gold standards for diagnosis of tuberculosis and drugresistance are Ziehl-Nielsen (acid-fast) stains and cultures. Although rapid culture systems (such as BACTEC) greatly reduce the 4-6 week waiting period for the slow-growing organism to form identifiable cultures, these systems are expensive and not practical in many of the high burden countries. Nucleic acid amplification tests (Su, 2002; Tenover, 2007; Daniel, 1990) and, more specifically, real-time PCR (Parashar et al., 2006) has long been recognized as a promising diagnostic tool for mycobacterial infections and antimicrobial resistance. Daniel (1990) mentioned the need for "rapid, simple and inexpensive" diagnostic methods, and when discussing PCR wrote that PCR "offers exciting promise," but also that "the technology is too complex for application in developing countries, but it has potential for simplification." Though great strides have been made in the development of nucleic acid amplification tests, it still cannot compete with the traditional methods on the basis of cost and simplicity of use, especially in developing countries. A systematic approach is needed to analyze each one of the steps: sample preparation, lysis of cells, DNA extraction, PCR amplification of targets, and the detection of PCR products. In this study, the kinetics of cell lysis is investigated.

DNA extraction/isolation is a key (and yet often overlooked) step in the molecular diagnostic process, and contributes a great deal to the overall sensitivity of an assay. The ideal nucleic acid isolation protocol will be simple and safe to perform, require minimal 
handling, use readily available equipment and involve inexpensive reagents. The isolation protocol should kill viable organisms rendering the sample safe for further handling and efficiently release nucleic acid material for amplification, all while incurring minimal damage to the genetic material. One must also consider the practicality/feasibility of the process. Many of the so-called high burden countries are developing nations with limited resources and have constraints on the type of techniques that can be successfully integrated into routine lab work (Daniel, 1990).

The development of a simple, effective lysis protocol hinges on a deeper understanding of the events occurring on a molecular level during chemical lysis. The bacterial cell wall is a complex, highly inter-linked system of polymers that ensures the structural integrity of the cell and allows the maintenance of proper turgor pressure. In order to gain access to the genetic material, the highly-crosslinked peptidoglycan structure of the cell wall must be disrupted. When the wall is weakened beyond a certain point, it can no longer withstand the pressure inside the cell. The weakened cell will rupture, thereby releasing the cellular contents including the genetic material.

As summarized by Garg et al. (2003), a wide variety of lysis protocols have been described: "sonication, boiling, SDS with lysozyme and heat, proteinase K, chaotropic salts, etc.". For example, sonication (combined with boiling and centrifugation) is the lysis method used in the FDA approved Gen-Probe amplified Mycobacterium tuberculosis direct test (AMTD) (Miller et al., 1994). Thomson et al. (2005) found that $20 \mathrm{~min}$ of boiling and a $5 \mathrm{~min}$ incubation at $55^{\circ} \mathrm{C}$ with microLYSIS ${ }^{\circledR}$ (Microzone Ltd., Haywards Heath, West Sussex) resulted in similarly sensitive PCR assays for detection of mycobacterial DNA. Kocagoz et al. (1993) concluded that three centrifugation washes followed by resuspension and $10 \mathrm{~min}$ of boiling in Tris-EDTA (TE) buffer ( $\mathrm{pH} 8.0$ ), resulted in better PCR amplification of TB genomic DNA than a sample treated with lysozyme, proteinase K and SDS followed by phenol-chloroform extraction and ethanol precipitation. Kocagoz et al. (2005) later reported successfully using a similar protocol (boiling for $20 \mathrm{~min}$ instead of $10 \mathrm{~min}$ ) to identify rifampin resistance in clinical isolates of TB using real-time PCR.

As stated by Kocagoz et al. (1993), the sample washes are necessary to remove proteins and salts produced during the decontamination step. Three washes effectively translate into three batches of supernatants that are discarded. However, Pathak et al. (2007) found that (1) supernatant of freshly processed samples (which is routinely discarded) contained mycobacterial DNA and (2) autolysis occurred during storage at low temperatures, leading to an increase in DNA in the supernatant; thus further highlighting the need for simplification of the lysis process, especially for patients with low bacterial loads and for analysis of stored samples.

In this work, the degree of lysis of M. smegmatis cells was measured as a function of time and temperature. Although there are important differences between $M$. smegmatis and M. tuberculosis (Hett and Rubin, 2008), M. smegmatis was chosen since it is a fastgrowing nonpathogenic species particularly useful in studying basic cellular processes of relevance to pathogenic mycobacteria. The M. smegmatis strain was transformed with green fluorescent protein (GFP) and provided high and uniform amounts of GFP expression and fluorescence upon excitation. As the opaque cells lysed and the protein was released into the buffer solution, an increase in fluorescence was observed. The rate of increase in the measured fluorescence is an indication of rate of lysis of the bacterial cells.

The experimental results are presented here along with two theoretical models. The first model is based on a Monte Carlo simulation of the lysis process and the accompanying probability density function (pdf), as described by the Fokker-Planck equation. The second model follows a chemical reaction engineering approach: the cell wall is modeled as layers, where each layer is made up of "blocks". Blocks can only be removed if they are exposed to the lysis solution and the model describes the rate of block exposure and removal.

\section{Materials and methods}

\subsection{M. smegmatis}

M. smegmatis was grown from stock $\mathrm{mc}^{2} 155$ (high-efficiency plasmid transformation mutant of M. smegmatis $\mathrm{mc}^{2} 6$ ) (Snapper et al., 1990). The $\mathrm{mc}^{2} 155$ strain was transformed by electroporation with the Escherichia-Mycobacterium multicopy $5.6 \mathrm{~kb}$ plasmid pBUN277. To construct plasmid pBUN277, a $1.4 \mathrm{~kb}$ fragment carrying the GFP gene under the control of the $M$. bovis BCG $h s p 60$ promoter was amplified from plasmid pWES4 (Parker and Bermudez, 1997) using primer pairs pwes4for (5'-GCA GCG AGG ACA ACT TGA G-3') and pwes4rev (5'-TTT CGA CTG AGC CTT TCG TT-3'). The amplified PCR fragment was ligated into vector pCR2.1, excised from this recombinant with HindIII, and cloned into the unique HindIII site of the E. coli-mycobacterium shuttle plasmid pMV206-H that carries a hygromycin-resistant marker (George et al., 1995). The construct with GFP gene transcription opposite to the transcription of oriM open reading frames (ORFs) was defined as pBUN277. Plasmids of this type, derived from the Mycobacterium fortuitum plasmid pAL500, replicate with a low copy number in mycobacteria (Stolt and Stoker, 1996).

M. smegmatis strains were grown at $37^{\circ} \mathrm{C}$ in Middlebrook $7 \mathrm{H} 9$ base broth supplemented with $0.5 \%$ bovine serum albumin fraction V (EM Science, Gibbstown, N.J.), 0.01 M dextrose (Sigma Chemical Co., St. Louis, Mo.), $0.015 \mathrm{M}$ sodium chloride and $0.2 \%$ glycerol (MADC) (as described by Chacon et al., 2002). The cultures were prepared using $20 \mathrm{~mL}$ of MADC and $100 \mu \mathrm{L}$ of glycerol-preserved stock. Liquid cultures were typically grown for $\sim$ $24 \mathrm{~h}$ at $37^{\circ} \mathrm{C}$ in an Innova 4300 rotary incubator (New Brunswick Scientific Co. Inc., Edison, N.J.) with shaking at $200 \mathrm{rpm}$. The culture was centrifuged at $2000 \mathrm{rpm}$ for $5 \mathrm{~min}$ at $4{ }^{\circ} \mathrm{C}$ in a TJ-6 centrifuge (Beckman Instruments, Inc., Fullerton, California, CA). The MADC supernatant was removed. A final cell concentration of $6 \times 10^{9}$ bacteria/mL was achieved by resuspension in TE buffer. Cell concentration was determined via optical density analysis using a Beckman DU-64 spectrophotometer at $\lambda=600 \mathrm{~nm}$. This concentration, $6 \times 10^{9}$ cells $/ \mathrm{mL}$, while very high, was useful in providing sufficient GFP signal.

\subsection{Lysis}

A $20 \mu \mathrm{L}$ volume of cell suspension was transferred to a microcentrifuge tube, centrifuged, supernatant discarded and resuspended in $20 \mu \mathrm{L}$ of lysis medium. A $5 \mu \mathrm{L}$ volume of cells in lysis medium was immediately transferred to a $20 \mu \mathrm{L}$ LightCycler $^{\circledR}$ capillary (Roche Applied Science, Indianapolis, IN) and subjected to analysis, to minimize the amount of premature lysis. The lysis media comprised different concentrations of TE buffer $(\mathrm{pH} 8.0$, $1 \mathrm{mM}$ EDTA and $10 \mathrm{mM}$ Tris- $\mathrm{HCl}$ for $1 \mathrm{X}$ buffer). A 1 min hold at $\sim 37^{\circ} \mathrm{C}$ ensured that each sample was at uniform starting conditions. The samples were then rapidly heated to the lysis temperature (heating time for the sample core was between 3.7 and $8.9 \mathrm{~s}$ depending on the lysis temperature). The core temperature of a representative glass capillary in the PCRJet ${ }^{\circledR}$ thermocycler is continually measured by a calibrated thermocouple. Trials were undertaken at $65,70,75,80,85$, and $90^{\circ} \mathrm{C}$.

Lysis was performed and tracked in a PCRJet ${ }^{\circledR}$ thermocycler $^{-1}$ (Megabase Research Products, Lincoln, NE). The sample was excited using a $470 \mathrm{~nm}$ blue LED (Model \# LS-450, Ocean Optics, Dunedin, FL) emitting light at $\lambda \sim 400-490 \mathrm{~nm}$, with a BG3 deep blue Schott filter (Edmund Optics, Barrington, NJ). GFP emission was read with a CCD-based spectrometer (Model \# USB-2000, 
Ocean Optics) at $\lambda=500-560 \mathrm{~nm}$. As the cells were lysed, GFP within the opaque cell wall was released into the lysis medium, thus increasing the spectrometer readout. Lysis progression was monitored by recording the spectrometer counts of the GFP emission over time at various temperatures.

\subsection{PCR amplification}

Presence of M. smegmatis DNA was confirmed with PCR amplification of a $451 \mathrm{bp} \mathrm{rpoB}$ gene target. Primers used were MS-rpoBF2 5 ' AGG TCG ACG ACA TCG ACC ACT TC 3', and MS-rpoBR2 5' TAC GGC GTC TCG ATG AAG CCG AAC 3'. Amplification was performed in a PCRJet ${ }^{\circledR}$ thermocycler (Megabase Research Products, Lincoln, NE), with $0.2 \mathrm{mM}$ of each dNTP, $5 \mathrm{mM} \mathrm{MgSO}_{4^{\prime}}$ $0.5 \mathrm{U}$ KOD Hot Start DNA Polymerase (Novagen, Madison, WI), 1X manufacturers buffer, $0.6 \mu \mathrm{M}$ of each primer, $10 \%$ (v/v) DMSO and $0.4 \mathrm{mg} / \mathrm{ml} \mathrm{BSA}$. To each reaction either $5 \mu \mathrm{L}$ of lysis product (before and after $80^{\circ} \mathrm{C}$ lysis), or $1 \mu \mathrm{L}$ of extracted purified genomic DNA was added as template. Cycling consisted of a $30 \mathrm{~s}$ hot start at $94^{\circ} \mathrm{C}$, followed by 35 cycles of $2 \mathrm{~s}$ denaturation at $94^{\circ} \mathrm{C}, 3 \mathrm{~s}$ annealing at $60^{\circ} \mathrm{C}$ and $3 \mathrm{~s}$ elongation at $72^{\circ} \mathrm{C}$.

\section{Results}

\subsection{GFP fluorescence measurements}

Results of the lysis experiments in 50X TE buffer, as described in Section 2.2, are shown in Figure 1 for temperatures between 65 and $90{ }^{\circ} \mathrm{C}$. The rate of lysis increases with temperature and no appreciable cell lysis occurred below $60^{\circ} \mathrm{C}$ (data not shown). As the M. smegmatis cells lyse, GFP is released and the fluorescence signal increases and reaches a local maximum before it slowly decreases. A gradual decrease in intensity after the fluorescence maximum has been reached can be ascribed to the denaturing of GFP in the lysis solution.

The GFP emission signals shown here have been normalized with respect to their maxima (original data files are available as Supplemental material). Experimental variances made this normalization necessary. These variances include differences in the optical properties of the individual LightCycler ${ }^{\circledR}$ capillaries, precise positioning of the capillary within the optical detection system of the PCRJet ${ }^{\circledR}$ thermocycler and interference with the optical detection by the opaque $M$. smegmatis cells at the bottom of the capillary. Together, these deviations created up to a $20 \%$ difference in the background signal with an approximately proportional difference in the optical signal maximums. While there were differences in sample-to-sample optical signals, no significant difference was seen in either the average background signal or the average maximum emission signals while adjusting the lysis conditions.

The plots shown in Figure 1 are representative results from single experiments. The outcome of GFP experiments depends on the bacterial culture composition (regarding cell wall thickness distribution) as mentioned in the text, as well as on GFP expression in the cells. These variations are difficult to control. By fitting the model to normalized data, the variation in GFP expression in the culture sample and the variation in absolute cell number can be addressed to a certain degree.

The variations in the initial distribution in cell wall thickness can be elucidated by fitting the model to replicates of the same experiment, thereby providing an average distribution. Any results would however be highly specific for the cultures being used in our study, and the application of the model to other cultures will most likely give different initial distributions. This fact only highlights the value of a generalized model in application to a broad range of organisms.
The maxima for the plots between 75 and $90^{\circ} \mathrm{C}$ lie very close. A subset of the cell population persists and more time is required to lyse these persisters at the lower temperatures. There is a further qualitative difference between the curves at 65 and $70^{\circ} \mathrm{C}$ compared to the curves at higher temperatures. At 65 and $70^{\circ} \mathrm{C}$ there is an initial steep rise in fluorescent output, followed by a near-plateau before a second steep increase occurs. A comparison between the curves at 65 and $70^{\circ} \mathrm{C}$ shows a decrease in the plateau length at the higher temperature and it disappears at temperatures above $70{ }^{\circ} \mathrm{C}$. This is an indication that the starting cell population is not uniform.

\section{2. $P C R$ results}

PCR amplification was used to confirm the DNA release from the M. smegmatis cells. Results of PCR amplification of a $451 \mathrm{bp}$ fragment of the rpoB gene of M. smegmatis (as described in Section 2.3) is shown in Figure 2. As a positive control, purified extracted DNA was used as template (Lane 2). Lane 3 shows amplification of the lysis solution before any thermal lysis treatment. As discussed in Section 1, some DNA is expected in the solution from natural autolysis of the cells, resulting in a weakly positive result. Lane 4 shows amplification of the lysis solution after thermal treatment and shows a stronger positive result. These results are not quantitative however; further studies are underway to confirm these results with real-time quantitative PCR.

\subsection{Mathematical modeling}

The $M$. smegmatis cell envelope structure includes a $\sim 7 \mathrm{~nm}$ plasma lipid bilayer membrane; two internal electron dense layers ( $\sim 4$ and $\sim 8 \mathrm{~nm}$, respectively) separated by a thin electrontransparent layer and an $\sim 8 \mathrm{~nm}$ outer membrane consisting of acyl lipids, mycolic acids, and porins (Hoffmann et al., 2008; Etienne et al., 2005). The individual internal layer compositions are not yet fully understood (Hoffmann et al., 2008), but they consist of peptidoglycan, arabinogalactan, and mycolate. The glycolipid, lipoarabinomannan, spans much of the cell envelope (Brennan and Nikaido, 1995). Since the precise structure and the physical and chemical properties of each individual layer is unknown, the cell wall envelope will be modeled as a pseudo-homogeneous structure with properties that are an average of the properties of each individual component. When such information becomes available, the models are amenable to these complexities.

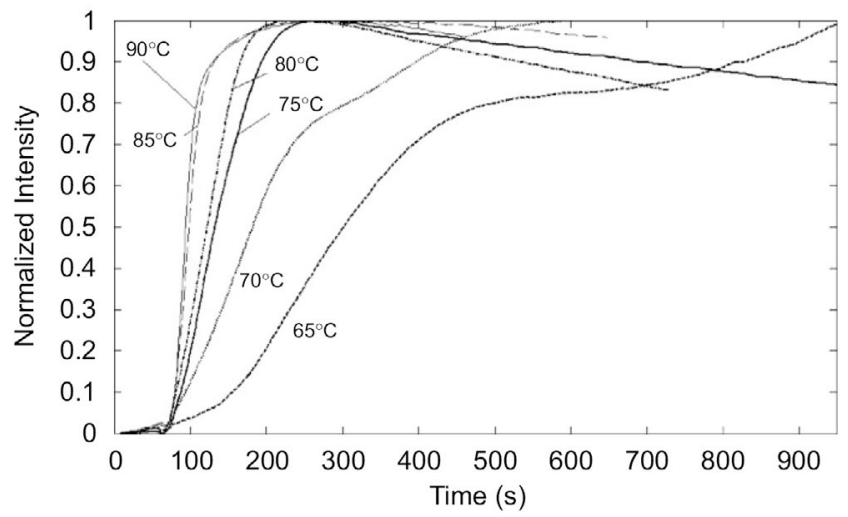

Figure 1. GFP fluorescence measurements over time at temperatures as indicated: (- - - - ) $65^{\circ} \mathrm{C}$; (...... ) $70^{\circ} \mathrm{C}$; ( $) 75^{\circ} \mathrm{C} ;(-\cdot . \cdot$ ) $80^{\circ} \mathrm{C}$; (- - - $85^{\circ} \mathrm{C}$; (- $) 90^{\circ} \mathrm{C}$. Fluorescence intensity measurements are normalized, each to their maximum value. The first $60 \mathrm{~s}$ of observations were at $\sim 37^{\circ} \mathrm{C}$, after which temperature is rapidly increased to the lysis temperature. 
A schematic of a section of the cell wall used for the model is shown in Figure 3. The cell wall in the model is divided into layers, labeled from 1 at the outer membrane to $M$ at the cytoplasmic membrane. Based on cryo-electron micrographs of cross sections of cells of M. smegmatis, the cell wall thickness (including both membranes, periplasmic layers, and periplasmic space) is estimated to have an average thickness of approximately $35 \mathrm{~nm}$, see supplemental Table 2 in Hoffmann et al. (2008). If $M=35$, then each layer is $1 \mathrm{~nm}$ in thickness. Every layer is discretized into $N$ blocks. Changes in the volume of blocks in inner layers compared to the outer layers, due to curvature at the poles, are neglected. Based on measurements for $M$. smegmatis cells (Greendyke et al., 2002; Dziadek et al., 2003), the average surface is estimated at $3 \mu \mathrm{m}^{2}$. If $N$ is set equal to $3 \times 10^{6}$, then the blocks are approximately cube shaped with $1 \mathrm{~nm}^{3}$ volumes.

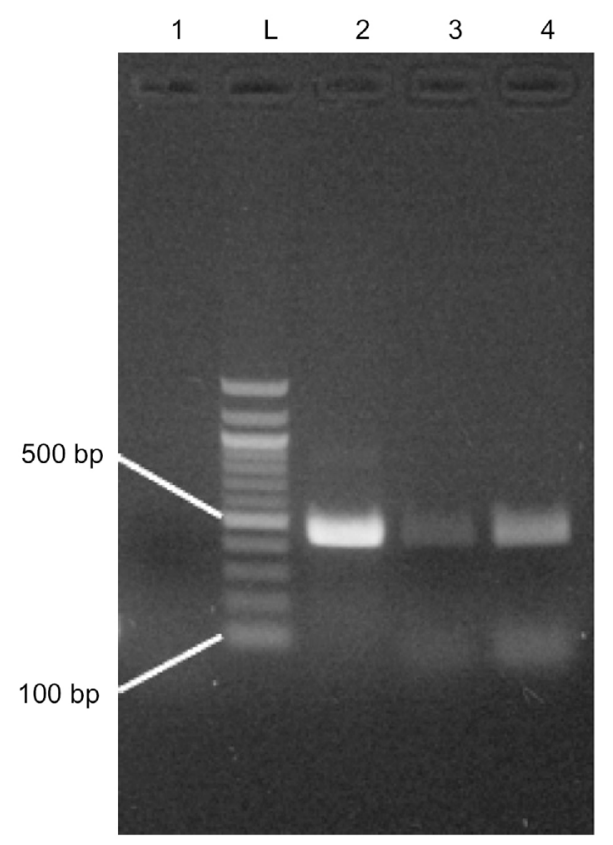

Figure 2. Gel electrophoresis results for amplification of lysed M. smegmatis cells. Lane 1 is a negative control (no template added), Lane 2 is a positive control (purified DNA as template), Lane 3 is amplification of pre-lysis M. smegmatis cells, and Lane 4 is amplification of post-lysis M. smegmatis cells.
Progressive chemical lysis from the outside of the cell surface due to the lysis buffer will be modeled. Although autolysins also contribute to cell lysis under physiological conditions, our experiments were carried out at temperatures well above temperatures at which autolysins would normally function. Therefore, autoysis should not adversely distort the lysis data. Autolysins target mostly older cell wall material (Pooley, 1976). Newly synthesized wall material is deposited underneath the inner surface of the older cell wall (Blümel et al., 1979). The picture that emerges from these observations is that autolytic activity is focused on the outer layers of the cell wall.

\subsubsection{Monte Carlo method}

The Monte Carlo method simulates the lysis of a single cell over time by randomly removing blocks which are exposed. A block $b_{i j}$ can be uniquely identified by the layer $j$ it belongs to ( 1 $\leq j \leq M$ ) and its position in that layer by an index $i$ (where $1 \leq i \leq$ $M)$. Only blocks with exposed faces are accessible to the lysing reagent. In 3-D models, the number of free faces of block $b_{i j}$ denoted by $\sigma_{i j}$, can vary between 0 and 5 . In 2 -D models, $0 \leq \sigma_{i j} \leq 3$. If $\sigma_{i j}=0$, then block $b_{i j}$ is either removed or it is still fully covered by other blocks. A Monte Carlo experiment consists of a large number of lysis events; each lysis event constitutes the removal of one block. At the onset of the $n$th lysis event in the Monte Carlo experiment, the total number of exposed faces is calculated:

$$
S(n)=\sum_{i=1}^{N} \sum_{j=1}^{35} \sigma_{i j}
$$

An exposed face is randomly selected from the pool of all exposed faces. Since an exposed face is uniquely associated with a block, that block is removed from the cell wall structure and the new set of exposed faces is calculated. Obviously blocks with a larger number of exposed faces are statistically favored for selection.

We assume that the initial number of free faces $S(1)=N$, i.e. a smooth starting cell surface. The $\mathrm{mc}^{2} 155$ M. smegmatis strain has a smooth surface; however, other M. smegmatis strains have a rough surface (Etienne et al., 2005). As the Monte Carlo simulation progresses, the cell surface becomes increasingly irregular. Thus, the number of exposed faces increases from $S(1)=$ $N$ until a quasi-steady state is reached. Only when blocks adjacent to the cytoplasm are removed (an unrealistic situation since cell rupture would occur prior to this), does $S$ decrease. In Figure 4 , the number of exposed edges $S(n)$ is plotted as a func- a

b

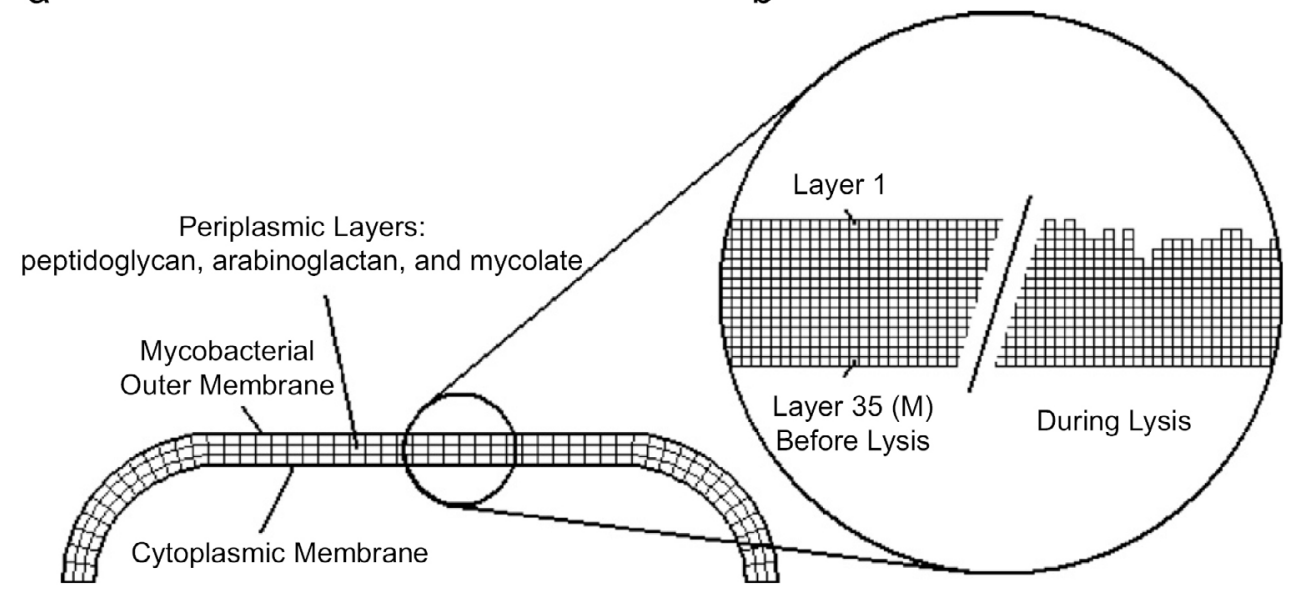

Figure 3. Two-dimensional cell envelope schematic: (a) M. smegmatis envelope with outer membranes and periplasmic structures and (b) model of the cell wall has uniform properties and a fine mesh for calculations. The mesh is shown before lysis and during lysis with some of the blocks removed. Note: for clarity, not all of the 35 layers are displayed. 


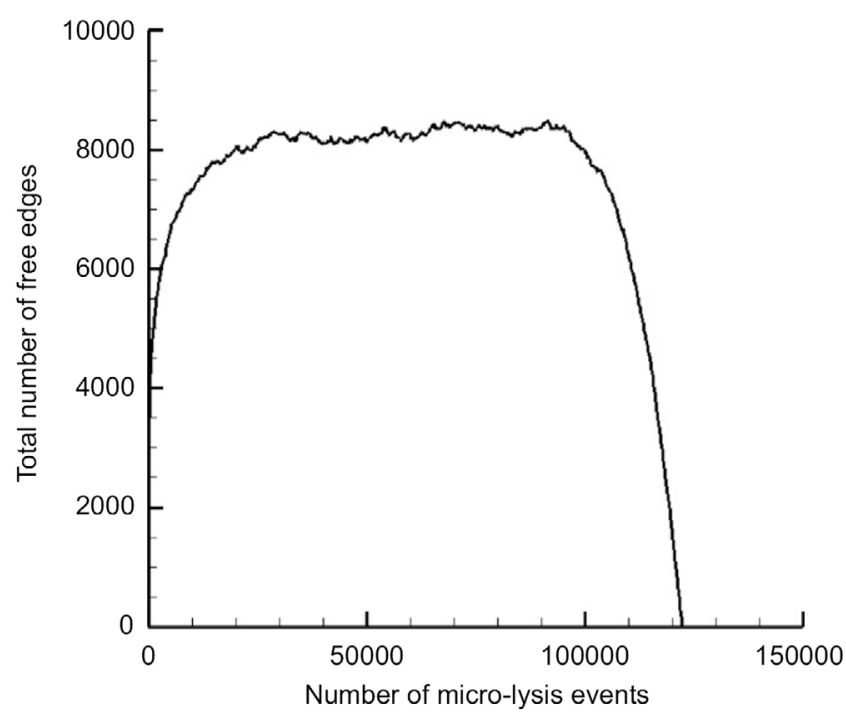

Figure 4. Number of free edges in a two-dimensional Monte Carlo simulation of the lysis of a cell wall that initially has 3500 free edges and consists of 35 layers.

tion of the lysis events $n$ for a 2-D Monte Carlo experiment with $N \times M=3500 \times 35$. At the onset, $S(1)=3500$ and $S$ reaches the quasi-steady state value of 8000 within 20000 lysis events. The rapid drop-off in $S$ for $n>100000$ is a result of blocks that are removed from the final layer. Interestingly, the average number of free edges/block during the quasi-steady state period is approximately 2.3 .

If the Monte Carlo experiment is repeated a large number of times, then the evolution (in terms of $n$ ) of the pdf, denoted as $F(n, m)$, can be calculated. The pdf $F(n, m)$ is defined as the probability to have an exposed face in layer $m$ at the onset of the $n$th lysis event. During the initial transient period where $S(n)$ increases, the pdf is not described by a binomial distribution because the probability, given by $1 / S(n)$, changes from one lysis event to the next. The pdf is given by a binomial distribution when $S$ is close to or at the quasi-steady state value $S$ $=S_{q s}$. The value of the Monte Carlo experiment with $N \times M=$ $3500 \times 35$ is to show that the quasi steady state is established quickly. Furthermore, the binomial distribution is well approximated by a normal distribution for large $n$; therefore, the pdf becomes

$$
F(n, m)=\frac{e^{-\left(m-1-n / S_{q s}\right)^{2} /\left(4 n / S_{q s}\right)}}{\sqrt{4 \pi n / S_{q s}}} .
$$

In Figure 5 the distribution of exposed faces $(F(n, m))$ for the 2-D case $N \times M=3500 \times 35$, is plotted as a function of position in the cell wall at intervals of 5000 lysis events. Layers are numbered from 1 at the outer surface to 35 at the inner surface of the cell wall, as described above. The pdf is calculated by repeating the Monte Carlo experiment 100000 times and averaging the results; it evolves from a delta function at $m=1$ to a binomial shape.

The Monte Carlo method is computationally expensive, but it has one advantage: the method easily accommodates changes in probability, i.e. changes in the number of exposed faces. The calculation of the realistic $3-\mathrm{D}$ case $N \times M=3,000,000 \times 35$ is prohibitively expensive. A strong argument can be made for the use of the Fokker-Planck model, which describes the evolution of the pdf through the layers.

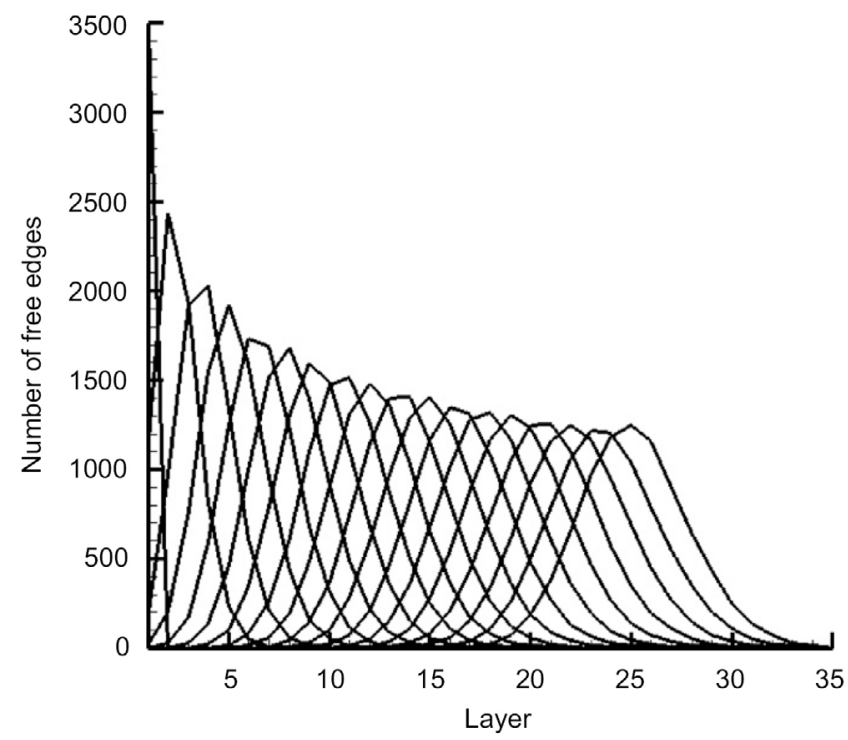

Figure 5. Progression of the pdf (not normalized) through cell wall layers, generated by the Monte Carlo method for a two-dimensional model that measures $N \times M=3,500 \times 35$.

\subsubsection{Fokker-Planck model}

The evolution of the normalized pdf as a function of time, on the domain $0 \leq x \leq M$ can be calculated directly from the FokkerPlanck equation:

$$
\partial f / \partial t=D \partial^{2} f / \partial x^{2}-V(t) \partial f / \partial x
$$

The function $f(t, x)$ in Equation (2) is analogous to $F(n, m)$ in Equation (1). The velocity $V$ changes until a quasi-steady state in the number of exposed faces is reached. However, the results of the Monte Carlo method show that the change is small and $D=V \approx$ $V_{q S}=k^{*} / S_{q S}$ can be used. The rate constant $k^{*}$ is assigned to the number of lysis events per second, thence $n=k^{*} t$. The solution that corresponds to an initial condition of $f(0, x)=\delta_{0}$ is

$$
f(t, x)=\frac{e^{-\left(x-k^{*} t / S_{q s}\right)^{2} /\left(4 k^{*} t / S_{q s}\right)}}{\sqrt{4 \pi k^{*} t / S_{q s}}}=\frac{e^{-(x-k t)^{2} /(4 k t)}}{\sqrt{4 \pi k t}},
$$

where $k=k^{*} / S_{g s}$ and $x=0$ corresponds to the first layer $m=1$. Also note that there exists a direct relationship between $n$ and $t$ as well as between $m$ and $x$, thus $F(m, n)$ and $f(t, x)$ holds equivalence.

To obtain a general solution, the initial condition $f(x, 0)=N \delta_{0}$ must be changed to include cells that are partially lysed. Let the initial state be given by $f(x, 0)=g(x)$, then the solution of Equation (2) is given by

$$
\begin{aligned}
& f(t, x)=\int \frac{g(s) e^{-(x-s-k t)^{2} /(4 k t)}}{\sqrt{4 \pi k t}} \mathrm{~d} s, \quad \text { for } x \geqslant s \text { and } \\
& f(t, x)=0 \text { for } x<s .
\end{aligned}
$$

To illustrate the effect of a distributed initial state, the following idealized two-peak distribution is used in Equation (4):

$$
g(x)=0.5 \delta_{0}+0.5 \delta_{15} .
$$

Thus $50 \%$ of the cells have wall thickness $M$ and $50 \%$ of the cells have wall thickness $M-15$. The distribution evolves from the initial two peaks to two normal distributions that overlap slightly at $V_{q s} t=k t / S_{q s}=4$, shown in Figure 6(a). At the later time $V_{q s} t=$ 10 (Figure 6(b)) the overlap increases and the combined curve is shown in blue. 

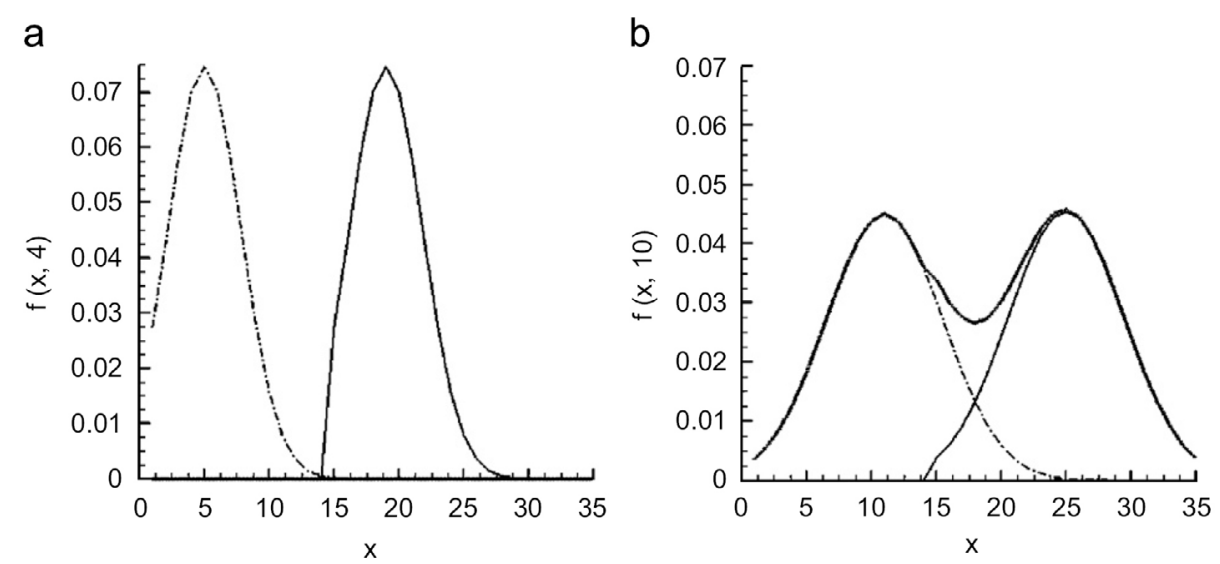

Figure 6. The progression of pdf $f(x, t)$ for initial data given by Equation (5). Shown at (a) $V_{q s} t=500$ and (b) $V_{q s} t=1000$. (- · - ·-) represents the $\delta_{0}$ population, and (-) represents the $\delta_{15}$ population at the indicated points in time. (.....-) in (B) represents the combined population.

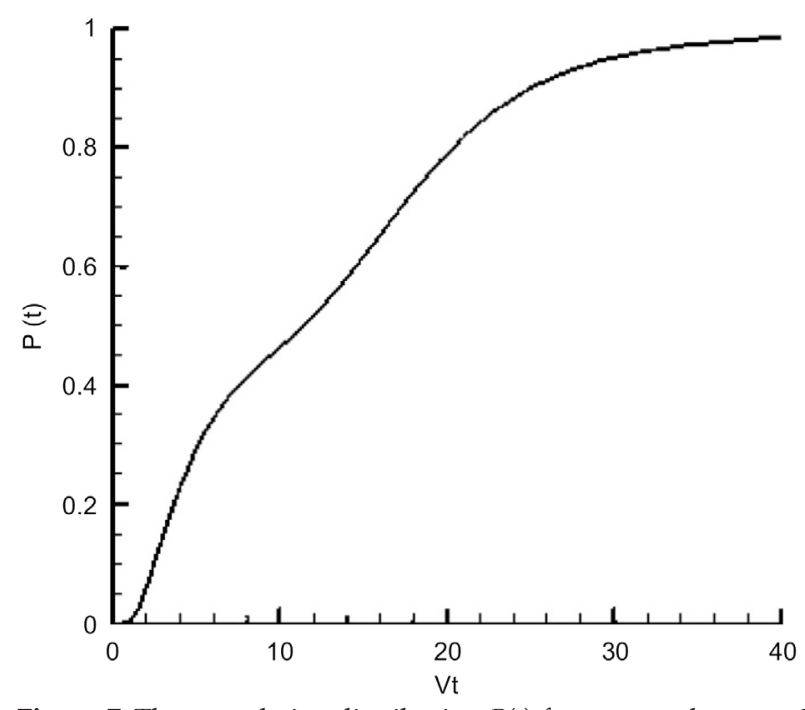

Figure 7. The cumulative distribution $P(t)$ for a case where $x=20$ and Equation (7) describes the initial population.

Turgor will cause the cell wall to rupture when a critical layer $m=m_{\text {crit }}$ is exposed. The critical layer is determined by fitting the theoretical models to the experimental results, see Section 3.4). Suppose the pdf with initial data (5) has $m_{\text {crit }}=20$ as the critical layer. The fraction of the cell population that has lysed as a function of time is given by the cumulative distribution:

$$
P(t)=\int_{m_{\text {crit }}}^{M} f(x, t) \mathrm{d} x
$$

In Figure 7 the cumulative distribution $P(t)$ is shown. Note the qualitative similarity between the curve in Figure 7 and the experimental curves at 65 and $70^{\circ} \mathrm{C}$ in Figure 1. It is the extent of separation between peaks that determine the extent of the plateau-like region in the curve. The overlap between peaks depends on the dispersion of the pdf; in the case of the experimental curves the dispersion depends on the rate constant $k$ and hence on the temperature. At higher temperatures the separate peaks of a multimodal initial distribution spread out faster and the multipeak character is lost much sooner than at lower temperaturesthis explains the differences between the experimental curves at lower temperature (observable plateaus) and higher temperature (no observable plateaus).
The initial data $g(s)$ in Equation (4) can be adjusted to fit the experimental data. Suppose the cell population is distributed evenly over the first four layers and the critical layer is $m_{\text {crit }}=6$. The expected outcome is that the $25 \%$ of cells that occupy layer $m=4$ will lyse first, followed by the cells in layer 3 and so forth. If the initial distribution occupies only a few layers adjacent to $m_{\text {crit' }}$ the GFP is released sooner and the lysis curve shifts toward shorter times, compared to an initial distribution that occupies more layers. The position of the inflection point in the lysis curve shifts right on the time axis if a larger fraction of cells is assigned to the outmost layer (furthest from $m_{\text {crit }}$ ). If the layers adjacent to $m_{c r i t}$ is more occupied, then the lysis curve may have no inflection point at all and the second derivative remains negative. Superimposed on the curvature is the effect of the rate constant. Larger (smaller) rate constants effectively shortens (lengthens) the time scale.

In Figure 8 the theoretical result (6) has been fitted to the experimental lysis curve for the 50X TE lysis buffer at $70^{\circ} \mathrm{C}$. The dotted and dashed curves present initial data that correspond to even distributions over 4 and 6 layers adjacent to $m_{c r i t}=6$, respectively. The initial distributions, denoted as $g_{4}$ and $g_{6}$, respectively, are:

$$
\begin{aligned}
& g_{4}(1 \ldots 6)=\{0 \% ; 0 \% ; 25 \% ; 25 \% ; 25 \% ; 25 \%\} \text { and } \\
& g_{6}(1 \ldots 6)=\{16.7 \% ; 16.7 \% ; 16.7 \% ; 16.7 \% ; 16.7 \% ; 16.7 \%\} .
\end{aligned}
$$

In both cases the curvature does not match the experimental curve - in Section 3.4 the number fraction that is assigned to each layer is adjusted to correct for this. However, the 6-layer model matches the experimental results better than the 4-layer model. (Although the results are not shown, other layers have also been compared). The 4-layer model will exhibit faster kinetics than the 6-layer model, because fewer layers need to be removed before lysis and this result is confirmed by a comparison of the dot curve (4-layer model) and dash curve (6-layer model) in Figure 8. The 6-layer model fits the experimental data best for $k=0.0204 \mathrm{~s}^{-}$ 1 . The best fitted results at the other temperatures are $k=0.0584 \mathrm{~s}^{-}$ ${ }^{1}$ at $80^{\circ} \mathrm{C}$ and $k=0.1213 \mathrm{~s}^{-1}$ at $90^{\circ} \mathrm{C}$.

\subsubsection{Multilayer model}

The multilayer model is introduced to offer an alternative and independent method to determine the rate constants and initial data. The model is also more intuitive to engineers. The cell wall is again modeled as $M$ consecutive layers and each layer consists of $N$ blocks. The layers are labeled as before, from 1 at the outer membrane to $m_{\text {crit }}$ the critical layer where cell wall failure occurs. Although there may be some variability in $m_{\text {crit' }}$ it is assumed in this study that $m_{\text {crit }}$ is constant. The value of $m_{\text {crit }}$ is determined 


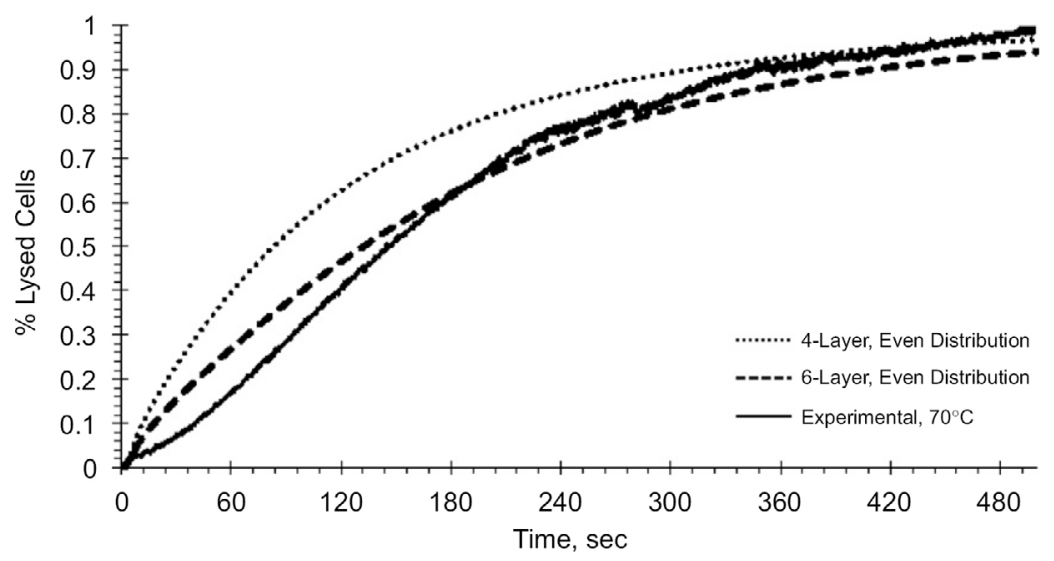

Figure 8. Results of the Fokker-Planck model with initial data of 6 evenly distributed layers $(-\ldots$ - $)$ and 4 evenly distributed layers (......) of exposed faces adjacent to $m_{\text {crit }}$. The experimental result for $70^{\circ} \mathrm{C}$ is also shown (—). The best fit is obtained for $k=0.0204 \mathrm{~s}^{-1}$. Note: the 4 -layer model is shifted to the left compared to the 6-layer model.

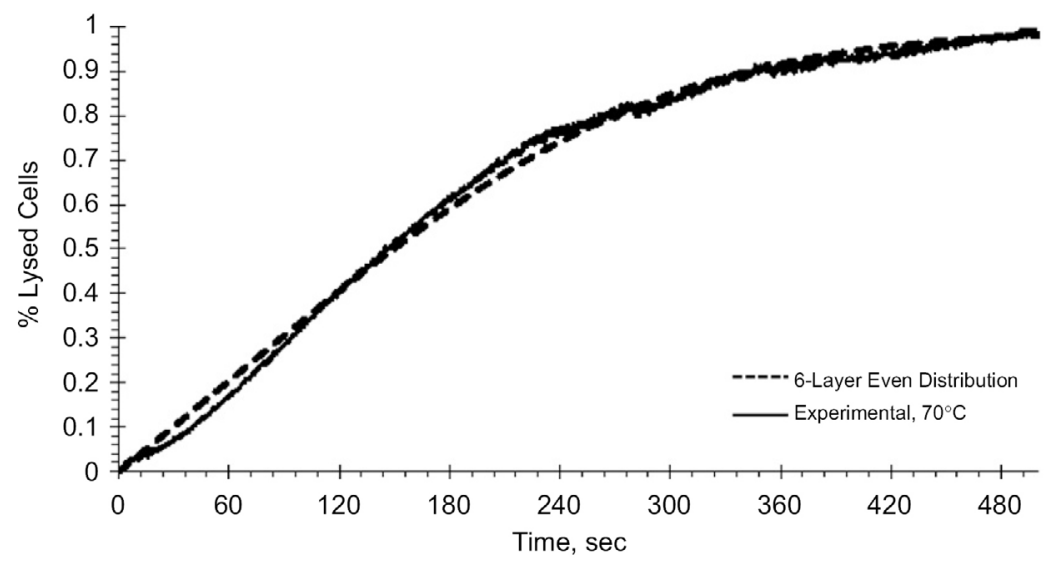

Figure 9. A comparison of the multilayer model result $\left(--{ }_{-}\right)$with initial data that corresponds to an even distribution over six layers and the experimental curve (—) at $70{ }^{\circ} \mathrm{C}$. The best fit is obtained for $k=0.0205 \mathrm{~s}^{-1}$.

by fitting the models to the experimental results-described in Section 3.4.

As already mentioned, the initial data depend on the variation in cell wall synthesis rates, variations in the autolysis activity from one cell to the other and the cessation of peptidoglycan synthesis in dead cells. If the sum of exposed blocks in the jth layer of all $C$ cells in the sample is denoted by $N_{j}^{\exp }(t)$, then the average number of exposed blocks in the $j$ th layer, expressed as a fraction is $A_{j}(t)=N_{j}^{\exp }(t) / N \times C$. The initial distribution is $g=\left\{A_{1}(0)\right.$, $\left.A_{2}(0), \ldots, A_{m c r i t}(0)\right\}$. Note that only layers at and above the critical layer are considered.

The blocks with exposed faces react with the lysing reagent. In the multilayer model we do not distinguish between blocks with different number of exposed faces. A balance over the outer layer gives

$$
\dot{A}_{1}=-k A_{1}
$$

The balancing equation for consecutive layers has the general form:

$$
\dot{A}_{j}=k A_{j-1}-k A_{j}, 2 \leq j \leq m .
$$

Note that removal of blocks in the layer $j-1$ exposes blocks in the $j$ th layer. For the initial distribution $g=\left\{A_{1}(0), A_{2}(0), \ldots, A_{m-}\right.$ crit $(0)\}$, the solution for the $j$ th layer is

$$
A_{j}(t)=e^{-k t} \sum_{i=1}^{j} \frac{A_{i}(0)(k t)^{j-i}}{(j-i) !} .
$$

The extent of lysis is then given by the number of blocks with exposed faces which have been removed from the critical layer (after normalizing by $N)$ :

$$
P(t)=\int_{0}^{t} k A m_{\text {crit }}\left(t^{\prime}\right) \mathrm{d} t^{\prime} .
$$

In Figure 9 the multilayer model (10) with initial data $A_{j}(0)=1 / 6$, for $1 \leq j \leq 6$ is compared to the experimental results at $70^{\circ} \mathrm{C}$ for the 50X TE buffer. The best fit of $k=0.0205 \mathrm{~s}^{-1}$ was obtained for the 6-layer model. The best fitted results at the other temperatures are $k=0.0577 \mathrm{~s}^{-1}$ at $80^{\circ} \mathrm{C}$ and $k=0.1218 \mathrm{~s}^{-1}$ at $90^{\circ} \mathrm{C}$.

\subsection{Discussion of results}

The best-fit values for $k$ at all three temperatures for the multilayer model match the best-fit results of the Fokker-Planck model closely (cf. Equations (4) and (6) and Equations (9) and (10)). This result is not surprising since the multilayer model and the Fokker-Planck (and therefore the Monte Carlo model) are orthogonal approaches with similar outcomes (ergodic theorem); the average lysis progression as given by a pdf that is obtained by repeated Monte Carlo experiments on a single cell is expected to be the 


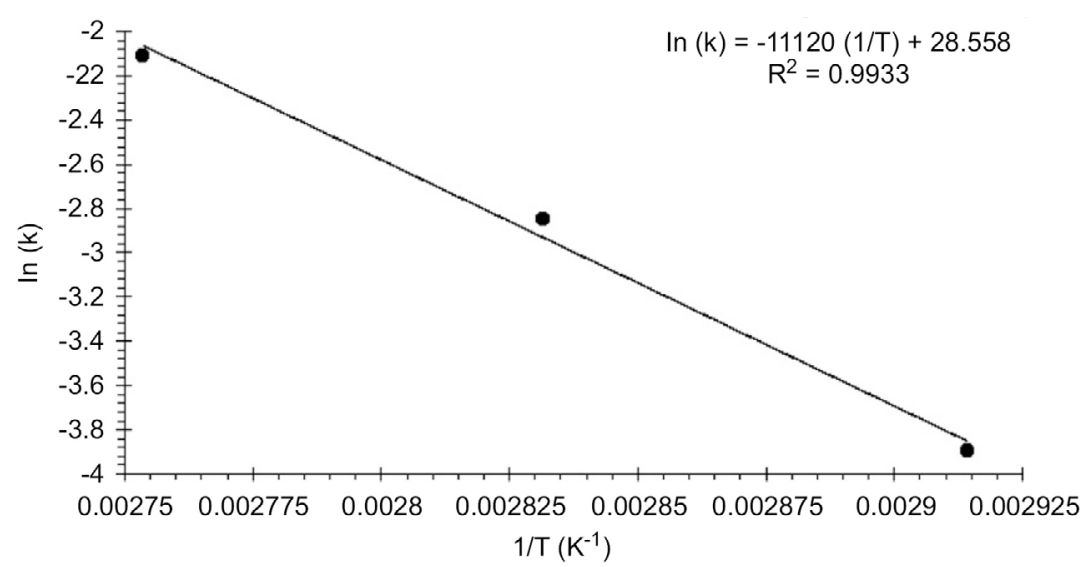

Figure 10. Arrhenius plot of average rate constants from Table 1. The activation energy is estimated to be $22.1 \mathrm{kcal} / \mathrm{mol}$.

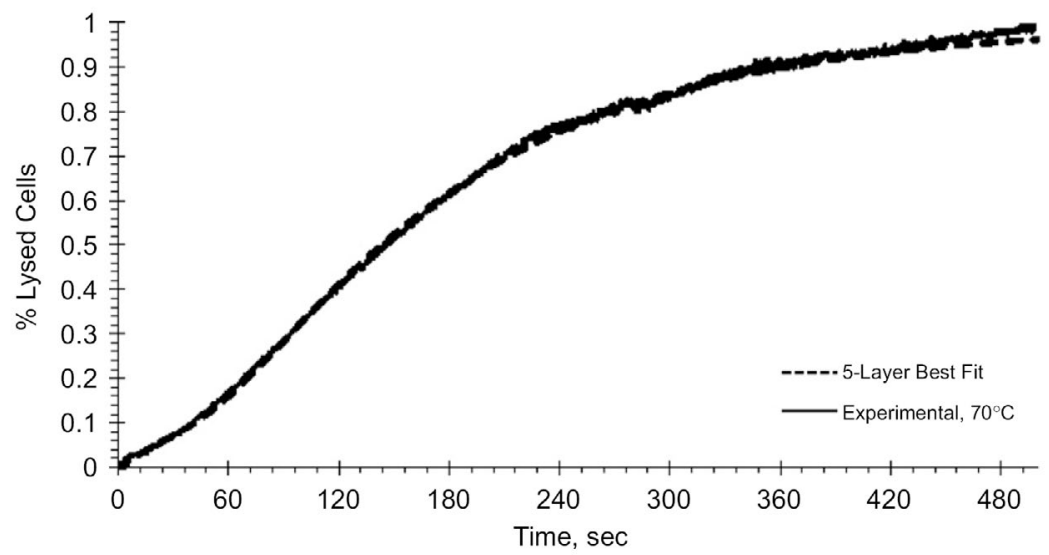

Figure 11. Improved fit of the multilayer model ( - - ) to experimental data at $70{ }^{\circ} \mathrm{C}(\longrightarrow-$ by adjusting the initial composition of the cell layers. The composition used here is: 78\% have five layers to break, $9 \%$ have three layers to break, $7 \%$ have two layers to break, and $6 \%$ have one layer to break for lysis to occur.

Table 1. Best fitted rate constants of the Fokker-Planck and multilayer models.

\begin{tabular}{lll}
\hline $\begin{array}{c}\text { Temper- } \\
\text { ature }\left({ }^{\circ} \mathrm{C}\right)\end{array}$ & $\begin{array}{l}\text { Fokker-Planck } \\
\text { model }\left(\mathrm{s}^{-1}\right)\end{array}$ & $\begin{array}{l}\text { Multilayer } \\
\text { model }\left(\mathrm{s}^{-1}\right)\end{array}$ \\
\hline 70 & 0.0204 & 0.0205 \\
80 & 0.0584 & 0.0577 \\
90 & 0.1213 & 0.1218 \\
\hline
\end{tabular}

same as the pdf obtained by averaging over many cells simultaneously. In Table 1 the rate constants are listed for the models.

An Arrhenius plot of the rate constants is shown in Figure 10. The activation energy is estimated to be $22.1 \mathrm{kcal} / \mathrm{mol}$. Unfortunately the temperature span that can be investigated is limited, but it is informative that the lysis reaction with the TE buffer slows down dramatically at lower temperatures. For example, at $50{ }^{\circ} \mathrm{C}$ the rate constant is $k=0.0028 \mathrm{~s}^{-1}$, which is nearly ten times less than at $70^{\circ} \mathrm{C}$.

It is possible, by adjusting the initial distribution of cells that occupy the different layers, to closely match the experimental and theoretical results. The best fit to the experimental results at $70{ }^{\circ} \mathrm{C}$ was obtained for $m_{\text {crit }}=5$ and

$$
g=\{78 \% ; 0 \% ; 9 \% ; 7 \% ; 6 \%\} .
$$

An $m_{\text {crit }}$ of 5 translates into $\sim 15 \%$ of the cell wall that is lysed before cell rupture. As a rough comparison, a mycolic-acid deficient mutant of corynebacterium glutamicum that has a $6.4 \mathrm{~nm}(\sim 16 \%)$ decreased wall thickness is a lethal mutation in M. smegmatis (Hoffmann et al., 2008). Note: the analysis for $70^{\circ} \mathrm{C}$ is shown here, but analysis of all of the experimental results shown in Figure 1 resulted in values of $m_{\text {crit }}$ corresponding to $14-17 \%$ of cell wall.

The best-fit value of the rate constant remained unchanged; $k=$ $0.020529 \mathrm{~s}^{-1}$. The results of the model using values given in Equation (11), are shown in Figure 11. The agreement between the experimental results and the theoretical model is excellent. If the initial data are adjusted for higher temperatures, then the bestfit results differ slightly from the initial data obtained at $70^{\circ} \mathrm{C}$, as in Equation (11). There may be several explanations for these differences, but one contributing factor is the difference in heat-up time to the operating temperature of the cuvette and small differences in the handling times of the cells/lysing buffer samples prior to heat-up.

In addition to changes in the chemical reaction of cell lysis, changes in concentration of the lysis buffer might result in altered osmotic pressure. The models do not explicitly account for osmotic pressure. However, the $m_{\text {crit }}$ values were empirically determined to best match the experimental findings. If the osmotic pressure does prove to be a factor: what we would expect for 
higher lysis buffer concentration is that if the rate constant $k$ is adjusted for the concentration and $m_{c r i t}=5$ is used to model the lysis, the theory would then predict slower lysis than what is observed experimentally. A downward adjustment of the mcrit value would then be necessary to more closely match the theory to experimental data, thereby showing agreement with increased osmotic pressure and less of the bacterial cell wall having to be removed to effect lysis.

\section{Conclusions}

The kinetics of the lysis reaction of $M$. smegmatis has been measured by the fluorescence of GFP that is released from lysed cells. A Monte Carlo has first been used to model the lysis reaction. Average behavior was obtained by multiple repeats of the Monte Carlo experiment, but the approach is too time-consuming to model the more realistic 3-D cases. Instead the FokkerPlanck equation, which describes the evolution of the pdf, has been solved and the solution has been fitted to the experimental data. The solution of the multilayer model has also been fitted to the experimental results and the rate constants from data-fitting, at different temperatures for the two models are very close. The rate constants have been used to calculate the activation energy for the reaction. Treating the initial data as unknown, the critical layer and initial state of lysis could be determined. The following conclusions can be made from this study:

- The activation energy for the lysis of M. smegmatis in TE buffer is $22.1 \mathrm{kcal} / \mathrm{mol}$ for a TE buffer strength of 50X.

- Based on fitted data, a typical cell lyses when its wall thickness is reduced at any position by more than $14-17 \%$ (estimated to be 5-6 $\mathrm{nm}$ for a thickness of $35 \mathrm{~nm}$ ).

- The models enable users to solve for the initial state of the cell wall-the initial state depends on the time the cells spend outside the culture medium, the temperature and the natural variation in cell wall synthesis and autolysis.

- Near complete lysis is accomplished in 90-200 s at lysis temperatures of 80 and $90^{\circ} \mathrm{C}$, respectively.

- This study provides valuable insight for the development of a rapid, simple lysis procedure for $M$. tuberculosis clinical samples to be tested via nucleic acid amplification tests.

\section{Acknowledgments}

Portions of this work were supported by Animal Health Project NEB14-141 (RGB, OC). We thank Drs. C. Barry and L. E. Bermudez for plasmids pMV206-H and pWES4, respectively. We thank D.K. Zinniel for assistance in transformation experiments and N. B. Harris for the construction of pBUN277.

\section{References}

Blümel et al., 1979 P. Blümel, W. Uecker and P. Giesbrecht, Zero order kinetics of cell wall turnover in Staphylococcus aureus, Arch. Microbiol. 121 (1979), pp. 103-110.

Brennan and Nikaido, 1995 P. J. Brennan and H. Nikaido, The envelope of mycobacteria, Аnnu. Rev. Biochem. 64 (1995), pp. 29-63.

Chacon et al., 2002 O. Chacon, Z. Feng, N. B. Harris, N. E. Caceres, L. G. Adams and R. G. Barletta, Mycobacterium smegmatis D-alanine racemase mutants are not dependent on D-alanine for growth, Antimicrob. Agents Chemother. 46 (2002), pp. 47-54.

Daniel, 1990 T. M. Daniel, The rapid diagnosis of tuberculosis: a selective review, J. Lab. Clin. Med. 116 (1990), pp. 277-282.
Dziadek et al., 2003 J. Dziadek, S. A. Rutherford, M. V. Madiraju, M. A. Atkinson and M. Rajagopalan, Conditional expression of Mycobacterium smegmatis ftsZ, an essential cell division gene, Microbiology 149 (2003), pp. 1593-1603.

Etienne et al., 2005 G. Etienne, F. Laval, C. Villeneuve, P. Dinadayala, A. Abouwarda, D. Zerbib, A. Galamba and M. Daffé, The cell envelope structure and properties of Mycobacterium smegmatis mc(2)155: is there a clue for the unique transformability of the strain?, Microbiology 151 (2005), pp. 2075-2086.

Garg et al., 2003 S. K. Garg, R. P. Tiwari, D. Tiwari, R. Singh, D. Malhotra, V. K. Ramnani, G. B. Prasad, R. Chandra, M. Fraziano and V. Colizzi et al., Diagnosis of tuberculosis: available technologies, limitations, and possibilities, J. Clin. Lab. Anal. 17 (2003), pp. 155-163.

George et al., $1995-$ K. M. George, Y. Yuan, D. R. Sherman and C. E. Barry 3rd, The biosynthesis of cyclopropanated mycolic acids in Mycobacterium tuberculosis. Identification and functional analysis of CMAS2, J. Biol. Chem. 270 (1995), pp. 27292-27298.

Greendyke et al., 2002 R. Greendyke, M. Rajagopalan, T. Parish and M. V. Madiraju, Conditional expression of Mycobacterium smegmatis dnaA, an essential DNA replication gene, Microbiology 148 (2002), pp. 3887-3900.

Hett and Rubin, 2008 E. C. Hett and E. J. Rubin, Bacterial growth and cell division: a mycobacterial perspective, Microbiol. Mol. Biol. Rev. 72 (2008), pp. 126-156.

Hoffmann et al., 2008 C. Hoffmann, A. Leis, M. Niederweis, J. M. Plitzko and $\mathrm{H}$. Engelhardt, Disclosure of the mycobacterial outer membrane: cryo-electron tomography and vitreous sections reveal the lipid bilayer structure, Proc. Natl. Acad. Sci. USA 105 (2008), pp. 3963-3967.

Kocagoz et al., 2005 T. Kocagoz, Z. Saribas and A. Alp, Rapid determination of rifampin resistance in clinical isolates of Mycobacterium tuberculosis by real-time PCR, J. Clin. Microbiol. 43 (2005), pp. 6015-6019.

Kocagoz et al., 1993 T. Kocagoz, E. Yilmaz, S. Ozkara, S. Kocagoz, M. Hayran, M. Sachedeva and H. F. Chambers, Detection of Mycobacterium tuberculosis in sputum samples by polymerase chain reaction using a simplified procedure, J. Clin. Microbiol. 31 (1993), pp. 1435-1438.

Miller et al., $1994-$ N. Miller, S. G. Hernandez and T. J. Cleary, Evaluation of gen-probe amplified Mycobacterium tuberculosis direct test and PCR for direct detection of Mycobacterium tuberculosis in clinical specimens, J. Clin. Microbiol. 32 (1994), pp. 393-397.

Parashar et al., $2006 \triangleright$ D. Parashar, D. S. Chauhan, V. D. Sharma and V. M. Katoch, Applications of real-time PCR technology to mycobacterial research, Indian J. Med. Res. 124 (2006), pp. 385-398.

Parker and Bermudez, 1997 A. E. Parker and L. E. Bermudez, Expression of the green fluorescent protein (GFP) in Mycobacterium avium as a tool to study the interaction between mycobacteria and host cells, Microb. Pathog. 22 (1997), pp. 193-198.

Pathak et al., 2007 D. Pathak, S. Chakravorty, M. Hanif and J. S. Tyagi, Lysis of tubercle bacilli in fresh and stored sputum specimens: implications for diagnosing tuberculosis in stored and paucibacillary specimens by PCR, BMC Microbiol. 7 (2007), pp. 83-92.

Pooley, 1976 H. M. Pooley, Turnover and spreading of old wall during surface growth of Bacillus subtilis, J Bacteriol 125 (1976), pp. 1127-1138.

Snapper et al., 1990 S. B. Snapper, R. E. Melton, S. Mustafa, T. Kieser and W. R. Jacobs Jr., Isolation and characterization of efficient plasmid transformation mutants of Mycobacterium smegmatis, Mol. Microbiol. 4 (1990), pp. 1911-1919.

Stolt and Stoker, 1996 P. Stolt and N. G. Stoker, Functional definition of regions necessary for replication and incompatibility in the Mycobacterium fortuitum plasmid pAL5000, Microbiology 142 (Pt 10) (1996), pp. 2795-2802.

Su, 2002 W. J. Su, Recent advances in the molecular diagnosis of tuberculosis, J. Microbiol. Immunol. Infect. 35 (2002), pp. 209-214.

Tenover, $2007 \checkmark$ F. C. Tenover, Rapid detection and identification of bacterial pathogens using novel molecular technologies: infection control and beyond, Clin. Infect. Dis. 44 (2007), pp. 418-423.

Thomson et al., 2005 L. M. Thomson, H. Traore, H. Yesilkaya, C. Doig, H. Steingrimsdottir, L. Garcia and K. J. Forbes, An extremely rapid and simple DNA-release method for detection of $M$. tuberculosis from clinical specimens, J. Microbiol. Methods 63 (2005), pp. 95-98. 